\title{
Effets des procédés de transformation sur la valeur nutritionnelle des formulations de bouillies de complément à base de sorgho
}

\author{
A. P. P. KAYODÉ ${ }^{*}$, F.U.G. AKOGOU, W. AMOUSSA HOUNKPATIN et \\ D. J. HOUNHOUIGAN \\ ${ }^{1}$ Département de nutrition et Sciences Alimentaires, Faculté des Sciences Agronomiques, \\ Université d'Abomey-Calavi, 01 BP 526 Cotonou, Bénin. \\ *Auteur correspondant; E-mail: polykap@yahoo.fr; Tel +22997870734
}

\section{RESUME}

La présente étude a évalué l'effet des procédés de transformation sur la valeur nutritionnelle de trois types de bouillies à base de sorgho. Il s'agit de: la bouillie de sorgho fermentée; la bouillie à base de sorgho, de soja et de fretin et la bouillie à base de sorgho, de niébé et d'arachide. Du point de vue nutritionnel, la bouillie de sorgho fermentée contient, sur la base sèche, $6,7 \%$ de protéines, $2,3 \%$ de lipides, et $1,17 \%$ de minéraux totaux. La bouillie à base de sorgho, de soja et de fretin contient $21 \%$ de protéines, $5,4 \%$ de lipides et $3,14 \%$ de minéraux totaux. La bouillie à base de sorgho, de niébé et d'arachide contient $17 \%$ de protéines, $0,98 \% \mathrm{~g}$ de lipides et 2,6\% de minéraux totaux. Le trempage des graines de sorgho pendant environ 23 heures, le tamisage après mouture et la fermentation ont entraîné une réduction significative $(\mathrm{P}<0,05)$ des teneurs en phénols totaux et en anthocyanes.

(C) 2012 International Formulae Group. All rights reserved.

Mots clés: aliment de complément, sorgho, valeur nutritionnelle, phénols, anthocyanes.

\section{INTRODUCTION}

Le sorgho (Sorghum bicolor L. Moench) est une graminée annuelle de la famille des Poaceae. C'est une culture importante dans les régions tropicales arides et sub-tropicales de l'Afrique, de l'Asie et de l'Amérique centrale (Kayodé, 2006). Naturellement résistant à la sécheresse, le sorgho assure la sécurité alimentaire et nutritionnelle de millions de personnes dans ces régions. Dans cette zone, il est cultivé pour ses grains destinés à la consommation humaine sous formes de pâtes, de bouillies et de boissons traditionnelles (Kayodé, 2006, Dicko et al., 2005). La farine de sorgho est mélangée dans différentes proportions pour améliorer la valeur nutritionnelle des bouillies traditionnelles (Nout et al., 1988). La malnutrition infanto-juvénile est un problème majeur de santé publique dans les pays en développement. Elle est la cause de la moitié des décès des enfants dans le monde (UNICEF, 2006). Près de 8,8 millions d'enfants meurent chaque année avant leur cinquième anniversaire (You et al., 2010) et les causes sont multiples. La malnutrition par carence en nutriments contribue pour $35 \%$ de ces décès (Black et al., 2008). La malnutrition par carence en nutriments chez les enfants désigne un ensemble de troubles 
caractérisés entre autres, par un arrêt ou un retard de la croissance et l'émaciation. L'utilisation d'aliments de complément de qualité nutritionnelle et sanitaire médiocre et la conduite inadéquate du processus du sevrage sont les facteurs qui exposent le plus les enfants aux carences en protéines, en énergie et aux carences en micronutriments (fer, zinc) (Latham, 2001; Sandstead, 2000; de Benoist, 1995).

Le Bénin à l'instar des autres pays en voie de développement est touché par ce fléau. L'étude sur l'analyse globale de vulnérabilité de la sécurité alimentaire et nutritionnelle (GdB et al., 2009) a révélé que quatre enfants sur dix souffrent de malnutrition chronique. Plus de $70 \%$ des enfants de moins de 5 ans souffrent d'anémie et les taux de retard de croissance linéaire varient de 25 à $40 \%$ chez les enfants d'âge scolaire au Bénin (Mitchikpè, 2007). Le département de l'Atacora compte parmi les régions les plus touchées par la malnutrition infantile au Bénin (INSAE, 2006). Dans certaines régions du Bénin comme l'Atacora, les céréales (maïs, sorgho, mil) contribuent pour $43 \%$ et $55 \%$ à la couverture des besoins énergétiques des enfants d'âge scolaire respectivement en période d'abondance et de soudure (Mitchikpè, 2007). La faible teneur de ces produits d'origine végétale en (micro)nutriments expliquent aussi les problèmes de carence en nutriments. Les polyphénols constituent un ensemble de molécules largement répandues dans le règne végétal. On les trouve dans la plante depuis les racines jusqu'aux fruits. Leur affinité pour les radicaux libres permet d'inhiber l'oxydation des lipoprotéines de faible densité, jouant ainsi un rôle positif dans la prévention des maladies cardiovasculaires. Cette propriété antioxydante est bénéfique et permet de prévenir la cancérogenèse (Hooper and Cassidy, 2006). Les polyphénols, notamment les formes condensées comme les tanins, ont également la faculté de complexer les cations $\left(\mathrm{Fe}^{2+}, \mathrm{Zn}^{2+}\right)$, réduisant ainsi leur biodisponibilité. Il existe peu d'études sur l'impact des opérations sur les composés bioactifs dans les aliments fermentés/maltés ou composés à base de sorgho. La présente étude a pour objectif d'évaluer l'effet des procédés technologiques de transformation de trois bouillies de complément à base de sorgho enrichies avec diverses sources protéiques, sur leurs valeurs nutritionnelles et les facteurs antinutritionnels.

\section{MATERIEL ET METHODES Matériel végétal}

Les grains de sorgho rouge [Sorghum bicolor], de légumineuses (soja[Glycine max], niébé [Vigna unguiculata], arachide [Arachis hypogaea]), et les fretins fumés utilisés dans cette étude ont été achetés sur le marché de Natitingou. Les fretins sont de petits vendus sous forme fumées. Ils sont moins chers et disponibles sur presque tous les marchés. Ces matières premières sont achetées chez diverses commerçantes et à différents jours de marché pour prendre en compte d'éventuelle variabilité dans leur composition. Les valeurs moyennes rapportées intègrent donc cette variabilité.

\section{Production et collecte des échantillons}

Chacun des trois aliments de complément a été transformé dans huit ménages à Natitingou au Nord-ouest du Bénin. Ces ménages ont été choisis de façon à diversifier leur statut socio-économique. Pour les bouillies de sorgho fermentées, cinq niveaux d'échantillonnage ont été définis. Il s'agit: des grains de sorgho nettoyés, les grains de sorgho trempés moulus, du produit moulu et tamisé, du produit tamisé fermenté et du produit fermenté cuit. Les bouillies composées ont été échantillonnées à trois niveaux. Il s'agit de: la farine composée, la bouillie dérivée après cuisson et la bouillie dérivée cuite après trempage. Après collecte, les produits secs (farines et graines) ont été stockés dans des sachets tandis que les produits liquides ou visqueux ont été stockés dans des pots. Les échantillons ont été 
conservés dans un congélateur pour être ensuite transportés au laboratoire. Tous les échantillons ont été séchés à l'étuve à $60^{\circ} \mathrm{C}$ pendant 24 heures pour être ensuite moulus au moulin Retsch (Retsch GmbH, Haan, Germany) muni d'un tamis de $1 \mathrm{~mm}$. Les farines ainsi obtenues sont utilisées pour les analyses physico-chimiques.

\section{Analyses physico-chimiques}

\section{Teneur en matière sèche}

$\mathrm{La}$ teneur en matière sèche des différents échantillons collectés, a été déterminée par séchage à l'étuve à $105{ }^{\circ} \mathrm{C}$ pendant 48 heures, suivie de pesée différentielle suivant la méthode AACC 4415A (AACC, 1984).

\section{Détermination du pH des bouillies}

La méthode utilisée est celle de Nout et al. (1989) modifiée. Environ $10 \mathrm{~g}$ de bouillie fermentée sont prélevés dans un bécher et additionnés de $20 \mathrm{ml}$ d'eau distillée. Le mélange est homogénéisé à l'aide de l'agitateur magnétique et le pH est mesuré. Le pH-mètre utilisé est étalonné avec les solutions tampons de $\mathrm{pH}=4$ et $\mathrm{pH}=7$. La valeur du $\mathrm{pH}$ est lue directement sur le cadran de l'appareil.

Dosage des lipides.

La méthode utilisée est celle de Soxlhet (AACC, 1984). Elle consiste à extraire pendant 4 heures les lipides libres de l'échantillon avec de l'éther de pétrole. L'extraction est suivie d'un séchage à l'étuve à $105^{\circ} \mathrm{C}$ pendant une heure les ballons sont refroidis au dessiccateur puis pesés. La teneur en lipides est exprimée en pourcentage sur la base sèche.

\section{Dosage des protéines brutes}

La méthode de Kjeldahl a été utilisée pour le dosage des protéines. Après minéralisation, distillation et titration, le taux de protéines brutes est déterminé à partir de la teneur en azote. La teneur en protéines est calculée en pourcentage sur la base sèche.

\section{Dosage des minéraux totaux}

Les minéraux totaux ou cendres brutes ont été déterminées par la méthode AACC 0801 (1984). La détermination est faite sur environ $5 \mathrm{~g}$ d'échantillon de farine. Après carbonisation, la farine est incinérée à $550^{\circ} \mathrm{C}$ pendant 24 heures. L'ensemble est à nouveau pesé après refroidissement dans un dessiccateur (en verre). Le taux de cendres est calculé en pourcentage de farine sèche.

Détermination de la teneur en anthocyanes

La teneur en anthocyanes des échantillons a été déterminée suivant la méthode spectrométrique modifiée (Abdel-Aal and Hucl, 1999). L'absorbance des extraits a été mesurée à $525 \mathrm{~nm}$ au moyen d'un spectrophotomètre (6715UV/Vis. Spectrphotometer, JENWAY). La concentration en anthocyanes a été déterminée et les résultats sont exprimés en $\mathrm{mg}$ pour $1 \mathrm{~g}$ d'échantillon (base sèche). La courbe de régression ayant servi à l'estimation de la concentration en anthocyane a été générée avec un coefficient de corrélation appréciable $\left(\mathrm{R}^{2}=0.9995\right)$. La cyanidine-3-glucoside a été utilisée comme standard.

Détermination de la teneur en phénols totaux

Les phénols totaux ont été mesurés par la méthode de Singleton et Rossi modifiée (Kayodé et al., 2007). A $300 \mu 1$ de chaque extrait ont été ajouté respectivement $4,2 \mathrm{ml}$ d'eau distillée, $0,75 \mathrm{ml}$ de réactif de FolinCiocalteu et $0,75 \mathrm{ml}$ de carbonate de sodium à $20 \%(\mathrm{P} / \mathrm{V})$. Les mélanges ont été homogénéisés après chaque ajout de réactif puis mis à l'incubation pendant 30 minutes. L'acide gallique a été utilisé comme standard.

\section{Analyses statistiques}

Les donnés ont été analysées avec les logiciels Excel et SPSS version 16. Le test d'ANOVA a été utilisé pour comparer les moyennes. Les moyennes de chacune des analyses physico-chimiques sont obtenues à partir de deux répétitions. Les données présentées sont les moyennes des huit répétitions. 


\section{RESULTATS}

Différents types de bouillies de complément à base de sorgho

Les bouillies à base de sorgho sont préparées avec divers types de matières premières. Les matières premières utilisées en combinaison avec le sorgho comprennent le maïs et le mil mais aussi des légumineuses comme le soja, le niébé et l'arachide. Occasionnellement, les fretins sont combinés à ces matières premières. Le trempage, la fermentation et la cuisson sont les principales opérations unitaires de transformation utilisées. Au cours de la préparation des bouillies de sorgho fermentées, les grains de sorgho sont nettoyés et trempés pendant 14 heures en moyenne. Les femmes effectuent ensuite une mouture au moyen du moulin à disque métallique de type $1^{\text {er }}$ A. Les grains trempés moulus sont délayés dans de l'eau pour subir un tamisage humide. Le produit obtenu à partir de ce tamisage est laissé fermenter pendant environ 18 heures. La bouillie de sorgho fermentée est enfin obtenue par une cuisson à environ $100{ }^{\circ} \mathrm{C}$ à une durée est d'environ 5 minutes. Deux types de farines composées ont été identifiés. Il s'agit de la farine composée I combinant les grains de sorgho et de soja et les fretins et la farine composée II combinant les grains de sorgho, niébé et arachide (Tableau 1). Pour la production des farines composées, chacun des ingrédients (grain) est trié, nettoyé et séché en tenant compte de sa proportion dans la farine composée. On procède ensuite à une torréfaction avant de faire le mélange des ingrédients. Ce mélange d'ingrédients est moulu pour obtenir la farine composée. Les farines composées sont soit cuites dans de l'eau pour en faire une bouillie ou parfois trempées dans de l'eau pendant environ 23 heures avant d'être cuites. La durée de cuisson des bouillies à base de farines composées (13 minutes) est significativement différente de celle des bouillies de sorgho fermentées (5 minutes).

\section{Caractéristiques nutritionnelles des différents types de bouillies}

La teneur en protéines des bouillies varie de 6,7 à 21,7\%. La bouille fermentée de sorgho a présenté la plus faible valeur et la bouille composée 1 (sorgho-soja-fretin) a présenté la plus forte valeur. La bouillie composée 2 (sorgho-niébé-arachide) a aussi présenté une teneur en protéines relativement élevée $(17,3 \%)$. Comparées à la bouillie ordinaire de sorgho, les bouillies enrichies avec les sources de protéines sont riches en protéines.

Il existe une différence significative entre les teneurs en minéraux totaux des différents types de bouillies. La bouillie de farine composée 1 (sorgho, soja et fretin) présente les valeurs en minéraux totaux les plus élevées $(3,14 \%)$. La bouillie de farine composée 2 (sorgho, niébé, et arachide) a une teneur en minéraux totaux significativement supérieure à celle de la bouillie de sorgho fermentée. La combinaison du sorgho avec des légumineuses et/ou des protéines animales aurait permis d'augmenter la teneur en minéraux totaux des bouillies dérivées. Le trempage des farines composées avant leur cuisson diminue de façon significative la teneur en minéraux totaux. Il y aurait donc eu perte de minéraux par diffusion dans l'eau de trempage.Dans les farines utilisées, les teneurs en phénols totaux sont de $9,88 \mathrm{mg} / \mathrm{g}, 11,03$ $\mathrm{mg} / \mathrm{g}$ et $14,29 \mathrm{mg} / \mathrm{g}$ respectivement dans la farine de sorgho, la farine composée 1 (sorgho, soja et fretin), et la farine composée 2 (sorgho, niébé et arachide) (Tableaux 3 et 4). L'analyse de variance a montré que le trempage des grains de sorgho, le tamisage et la fermentation réduisent de façon significative les teneurs en phénols totaux et en anthocyanes $(\mathrm{P}<0,05)$. Le pourcentage de réduction après trempage est respectivement de $31 \%$ et de $41 \%$ pour les phénols totaux et les anthocyanes. Le tamisage réduit les phénols et les anthocyanes de 33\% et $27 \%$ respectivement. A la suite de la fermentation on observe une réduction des 
phénols et des anthocyanes respectivement de $41,7 \%$ et de $86 \%$. De l'analyse des Tableaux 4 et 5 , il ressort que le trempage des deux types de farines composées avant cuisson a un effet significatif sur la réduction des teneurs en phénols totaux et en anthocyanes. En effet, suite au trempage, on observe une réduction des phénols totaux et des anthocyanes respectivement de $40 \%$ et de $87 \%$ dans le cas de la bouillie de farine composé I (sorgho, soja, fretin). Les phénols et les anthocyanes ont été réduits respectivement de $19 \%$ et de $75 \%$ dans le cas des bouillies de sorgho, de niébé et d'arachide. Au cours du trempage, il y aurait donc eu une fermentation permettant la production des polyphénolases par les microorganismes. Les variations de $\mathrm{pH}$ (6 à 4,5) notées au cours du trempage desdites farines composées attestent de cette fermentation (Tableau 2).

Tableau 1: Différents types de bouillies étudiées et leur mode de préparation.

\begin{tabular}{|c|c|c|c|}
\hline Plats & $\begin{array}{l}\text { Matières premières et } \\
\text { ingrédients }\end{array}$ & $\begin{array}{l}\text { Opérations } \\
\text { unitaires } \\
\text { impliquées }\end{array}$ & Description de l'opération \\
\hline \multirow{5}{*}{$\begin{array}{l}\text { Bouillie } \\
\text { fermentée } \\
\text { de sorgho }\end{array}$} & \multirow{5}{*}{ Grains de Sorgho rouges $(100 \%)$} & trempage & $\begin{array}{c}\text { Dans de l'eau à température } \\
\text { ambiante pendant } 14 \mathrm{~h}\end{array}$ \\
\hline & & mouture & $\begin{array}{l}\text { A l'aide d'un moulin à disque } \\
\text { de type } 1^{\text {er }} \mathrm{A}\end{array}$ \\
\hline & & tamisage & $\begin{array}{c}\text { A l'aide de tissu de maille } \\
\text { d'environ } 1 \mathrm{~mm}\end{array}$ \\
\hline & & fermentation & $\begin{array}{l}\text { Dans de l'eau à température } \\
\text { ambiante pendant environ } 18 \mathrm{~h}\end{array}$ \\
\hline & & cuisson & $\begin{array}{l}\text { Au feu de bois pendant environ } \\
10 \mathrm{~min}\end{array}$ \\
\hline \multirow{5}{*}{$\begin{array}{l}\text { Bouillie de } \\
\text { farine } \\
\text { composée I }\end{array}$} & \multirow{5}{*}{$\begin{array}{l}\text { - Grain de sorgho (61 à } 69 \% \text { ) } \\
\text { - Grains de soja (27 à 35\%) } \\
\text { - Fretin (2 à 6\%) }\end{array}$} & lavage & $\begin{array}{c}\text { Dans de l'eau à température } \\
\text { ambiante }\end{array}$ \\
\hline & & séchage & $\begin{array}{c}\text { Les ingrédients sont séchés au } \\
\text { soleil }\end{array}$ \\
\hline & & torréfaction & $\begin{array}{c}\text { Les ingrédients sont grillés } \\
\text { séparément }\end{array}$ \\
\hline & & mélange & $\begin{array}{l}\text { Les ingrédients sont mis } \\
\text { ensemble dans un récipient }\end{array}$ \\
\hline & & mouture & $\begin{array}{l}\text { A l'aide d'un moulin à disques } \\
\text { de type } 1^{\text {er }} \mathrm{A}\end{array}$ \\
\hline \multirow{5}{*}{$\begin{array}{l}\text { Bouillie de } \\
\text { farine } \\
\text { composée } \\
\text { II }\end{array}$} & \multirow{5}{*}{$\begin{array}{l}\text { - Sorgho (45 à } 56 \% \text { ) } \\
\text { - Niébé (42 à 51\%) } \\
\text { - Arachide (2 à 4\%) }\end{array}$} & lavage & $\begin{array}{c}\text { Dans de l'eau à température } \\
\text { ambiante }\end{array}$ \\
\hline & & séchage & $\begin{array}{c}\text { Les ingrédients sont séchés au } \\
\text { soleil }\end{array}$ \\
\hline & & torréfaction & $\begin{array}{c}\text { Les ingrédients sont grillés } \\
\text { séparément }\end{array}$ \\
\hline & & mélange & $\begin{array}{l}\text { Les ingrédients sont mis } \\
\text { ensemble dans un récipient }\end{array}$ \\
\hline & & mouture & $\begin{array}{l}\text { A l'aide d'un moulin à disques } \\
\text { de type } 1^{\text {er }} \mathrm{A}\end{array}$ \\
\hline
\end{tabular}


Tableau 2: Composition proximale des bouillies.

\begin{tabular}{|c|c|c|c|c|}
\hline Aliments & $\begin{array}{l}\text { Protéines } \\
(\% \mathrm{MS})\end{array}$ & $\begin{array}{l}\text { Lipides } \\
\text { (\% MS) }\end{array}$ & $\begin{array}{c}\text { Minéraux } \\
\text { totaux } \\
(\% \mathrm{MS})\end{array}$ & pH \\
\hline $\begin{array}{l}\text { Bouillie fermentée de sorgho } \\
(\mathrm{n}=8)^{*}\end{array}$ & $6,74 \pm 0,74^{\beta} \mathrm{a}$ & $2,34 \pm 0,90 \mathrm{a}$ & $1,18 \pm 0,36 \mathrm{a}$ & $3,74 \pm 0,14 a$ \\
\hline $\begin{array}{l}\text { Bouillie composée } 1 \text { (sorgho-soja- } \\
\text { fretin) }(n=8)\end{array}$ & $21,66 \pm 2,34 b$ & $5,42 \pm 1,13 b$ & $3,14 \pm 0,39 b$ & $5,99 \pm 0,42 b$ \\
\hline $\begin{array}{l}\text { Bouillie composée } 1 \text { trempée }(n= \\
\text { 8) }\end{array}$ & $21,02 \pm 2,43 b$ & $4,45 \pm 0,88 \mathrm{c}$ & $2,93 \pm 0,37 b, d$ & $4,47 \pm 0,17 \mathrm{c}$ \\
\hline $\begin{array}{l}\text { Bouillie composée } 2 \text { (sorgho- } \\
\text { niébé-arachide) }(n=8)\end{array}$ & $17,25 \pm 0,79 \mathrm{c}$ & $0,99 \pm 0,20 \mathrm{~d}$ & $2,64 \pm 0,18 \mathrm{c}$ & $5,87 \pm 0,35 b$ \\
\hline $\begin{array}{l}\text { Bouillie composée } 2 \text { trempée } \\
(n=8)\end{array}$ & $16,95 \pm 0,86 \mathrm{c}$ & $0,85 \pm 0,18 d$ & $2,47 \pm 0,50 c, d$ & $4,70 \pm 0,23 c$ \\
\hline
\end{tabular}

Tableau 3: Influence des opérations unitaires de transformation sur les teneurs en phénols totaux et en anthocyanes de la bouillie fermentée à base de sorgho.

\begin{tabular}{|c|c|c|c|c|c|}
\hline & $\begin{array}{l}\text { Grains } \\
(\mathrm{n}=8) *\end{array}$ & $\begin{array}{c}\text { Grains trempés } \\
\text { moulus } \\
(n=8)\end{array}$ & $\begin{array}{l}\text { Grains moulus } \\
\text { tamisés }(n=8)\end{array}$ & $\begin{array}{c}\text { Grains } \\
\text { tamisés } \\
\text { fermentés } \\
(n=8)\end{array}$ & $\begin{array}{c}\text { Produit } \\
\text { fermenté } \\
\text { cuit } \\
(n=8)\end{array}$ \\
\hline $\begin{array}{l}\text { Phénols totaux } \\
\text { (mg/g MS) }\end{array}$ & $9,88 \pm 1,64^{\beta} \mathrm{a}$ & $6,72 \pm 1,82 b$ & $4,46 \pm 1,40 \mathrm{c}$ & $6,32 \pm 1,50 \mathrm{~d}$ & $2,12 \pm 0,53 \mathrm{e}$ \\
\hline $\begin{array}{l}\text { Anthocyanes } \\
(\mathrm{mg} / \mathrm{g} \text { MS) }\end{array}$ & $0,24 \pm 0,09 \mathrm{a}$ & $0,14 \pm 0,07 b$ & $0,102 \pm 0,05 b$ & $0,19 \pm 0,08 \mathrm{c}$ & $0,02 \pm 0,02 d$ \\
\hline
\end{tabular}

Tableau 4: Influence des opérations unitaires de transformation sur les teneurs en phénols totaux et en anthocyanes de la bouillie de farine composée à base de sorgho, de soja et de fretin.

\begin{tabular}{|c|c|c|c|}
\hline & $\begin{array}{c}\text { Farine composée } \\
\text { sorgho-soja-fretin } \\
\text { brute } \\
(\mathrm{n}=8)^{*}\end{array}$ & $\begin{array}{c}\text { Farine composée } \\
\text { sorgho-soja-fretin } \\
\text { cuite } \\
(n=8)\end{array}$ & $\begin{array}{c}\text { Farine composée } \\
\text { sorgho-soja-fretin } \\
\text { trempée et cuite } \\
(n=8)\end{array}$ \\
\hline $\begin{array}{l}\text { Phénols totaux } \\
\text { (mg/g MS) }\end{array}$ & $11,03 \pm 0,6^{\beta} a$ & $6,29 \pm 0,62 b$ & $8,74 \pm 0,42 c$ \\
\hline $\begin{array}{l}\text { Anthocyanes } \\
(\mathrm{mg} / \mathrm{g} \mathrm{MS})\end{array}$ & $0,122 \pm 0,03 \mathrm{a}$ & $0,039 \pm 0,01 b$ & $0,073 \pm 0,00 \mathrm{c}$ \\
\hline
\end{tabular}


Tableau 5: Influence des opérations unitaires de transformation sur les teneurs en phénols totaux et en anthocyanes de la bouillie de farine composée à base de sorgho, niébé et d'arachide.

\begin{tabular}{|c|c|c|c|}
\hline & $\begin{array}{l}\text { Farine composée sorgho- } \\
\text { niébé-arachide bute } \\
\qquad(\mathrm{n}=8)^{*}\end{array}$ & $\begin{array}{c}\text { Farine } \\
\text { composée } \\
\text { sorgho-niébé- } \\
\text { arachide cuite } \\
\quad(n=8)\end{array}$ & $\begin{array}{c}\text { Farine } \\
\text { composée } \\
\text { sorgho-niébé- } \\
\text { arachide } \\
\text { trempée et cuite } \\
(n=8)\end{array}$ \\
\hline $\begin{array}{l}\text { Phénols totaux } \\
(\mathrm{mg} / \mathrm{g} \text { MS })\end{array}$ & $14,29 \pm 0,95^{\beta} \mathrm{a}$ & $4,51 \pm 0,73 b$ & $5,38 \pm 0,51 \mathrm{c}$ \\
\hline Anthocyanes (mg/g MS) & $0,16 \pm 0,04 \mathrm{a}$ & $0,04 \pm 0,01 b$ & $0,07 \pm 0,01 \mathrm{c}$ \\
\hline
\end{tabular}

\section{DISCUSSION}

La teneur en protéines des aliments de complément destinés aux enfants de la tranche d'âge de 6 à 35 mois doit être supérieure ou égale à $15 \mathrm{~g} / 100 \mathrm{~g}$ de matière sèche (FAO/OMS, 1994). Le Codex Alimentarius (FAO/WHO, 2002) précise aussi que la teneur en protéines de ce type d'aliment ne doit pas dépasser 22,6 g/100 g de matière sèche. De l'analyse de nos données, il ressort que la bouillie de sorgho fermentée n'a pas la teneur en protéines requise pour servir d'aliments de complément. Par contre, les bouillies de farines composées répondent tout à fait à ce critère (Tableau 2). La plus forte teneur en protéines a été obtenue dans le cas de la bouillie de farine composée à base de sorgho, de soja et de fretin $(21,7 \%)$. Ceci pourrait s'expliquer par la qualité des ingrédients qui composent ce type de bouillie. Les fretins et le soja, deux ingrédients reconnus pour leur richesse en protéines seraient les principaux constituants qui confèrent à cette bouillie sa richesse en protéines. De plus, l'usage simultané de protéines d'origine animale (fretin) et de protéines d'origine végétale (soja) constitue une pratique alimentaire intéressante pour l'alimentation des enfants de 6 à 35 mois. En effet, l'utilisation des protéines d'origine animales favorise l'absorption des minéraux comme le fer (Lestienne, 2004). Malgré que sa teneur en protéines soit inférieure à celle de la bouillie de farine composée à base de sorgho, de soja et de fretin, la bouillie de farine composée à base de sorgho, de niébé et d'arachide répond aussi au critère nutritionnel concernant les apports recommandés en protéines pour la tranche d'âge de 6 à 35 mois. Elle constitue donc une bonne alternative à la bouillie de farine composée à base de sorgho, de soja et de fretin. Du point de vue de l'impact des opérations unitaires de transformation, il est apparu que le trempage des farines composées avant leur cuisson n'a pas eu d'effet significatif sur leur teneur en protéines. Par contre, cette opération favorise l'abaissement du $\mathrm{pH}$ permettant ainsi d'inhiber le développement de la majorité des microorganismes pathogènes (Nout, 1994). Il serait donc plus intéressant qu'au sein des ménages, la pratique de trempage soit encouragée.

Pour la tranche d'âge ciblée (6 à 35 mois), la teneur en lipides de l'aliment de complément doit être comprise entre 12 et $25 \%$ de matière sèche (FAO/OMS, 1994). Toutes les bouillies étudiées présentent une teneur en lipides de l'ordre de $0,85 \mathrm{~g}$ à $5,42 \mathrm{~g}$ pour $100 \mathrm{~g}$ de matière sèche (Tableau 2). La différence entre les teneurs en lipides des bouillies fermentées à base de sorgho, des bouillies de farine composée 1 (sorgho, soja et fretin), et des bouillies de farine composée2 (sorgho, niébé 
et arachide) est significative $(\mathrm{P}<0,05)$. La bouillie de farine composée à base de sorgho, de soja et de fretin a la teneur en lipides la plus élevée $(5,4 \%)$. La teneur en lipide la plus faible est celle des bouillies composées à base de sorgho, de niébé et d'arachide (0,85\%).Selon Lestienne (2004), la composition en lipides des graines de niébé est faible, de l'ordre de 1,2\%. De plus, le niébé se trouve en forte proportion dans le mélange sorgho-niébé-arachide (42 à 51\%). Ceci pourrait expliquer la faible teneur en lipides des bouillies de farine composée à base de sorgho, de niébé et d'arachide. Le trempage de la farine avant cuisson a réduit de façon significative $(\mathrm{P}<0,05)$ la teneur en lipides des bouillies de farine composée 1 (sorgho, soja et fretin). Pour ce qui concerne les minéraux, il a été rapporté que l'usage des protéines animales pourrait favoriser l'absorption de certains minéraux comme le fer (Lestienne, 2004). De ce point de vue, la farine composée I contenant les fretins restent particulièrement intéressante. La bioactivité des composés phénoliques réside dans leur capacité antioxydante favorable à la prévention des maladies chroniques. Dans ce contexte, nous avons évalué l'effet des opérations unitaires de transformation sur un certain nombre de constituants bioactifs, notamment les composés phénoliques dont les phénols totaux et les anthocyanes. Divers travaux de recherche ont démontré la corrélation entre la concentration en composés phénoliques des aliments et leur capacité antioxydante. Ainsi, il existe une forte corrélation $(r=0,95)$ entre les phénols totaux et le pouvoir antioxydant dans la fraise (Tulipani et al. 2008). De même Podsędek et al. (2008) ont trouvé une forte corrélation entre les anthocyanes et l'activité antioxydante dans le chou rouge. Le trempage des grains de sorgho permet de les débarrasser des enveloppes comme les glumes qui sont riches en composés phénoliques (Kayodé et al., 2007). Au cours du tamisage, le son est éliminé réduisant davantage les taux de phénols totaux. Au cours de la fermentation, la production des polyphénoloxydases aurait entraîné la dégradation des composés phénoliques complexe comme les tanins (Kayodé, 2006; Lestienne, 2004). L'augmentation de la teneur en composés phénoliques après fermentation s'explique par le fait que la méthode utilisée mesure les groupements hydroxyles. La fermentation favorise une dépolymérisation des molécules phénoliques condensées entraînant une augmentation des groupements hydroxyles mesurés (Kayodé et al., 2007). La cuisson a un effet contraire sur les composés phénoliques. Elle favorise la polymérisation des composés phénoliques. Ceci réduit donc le nombre de groupements hydroxyles mesurés.

\section{Implications nutritionnelles des résultats}

La composition nutritionnelle de la bouillie de sorgho fermentée fait d'elle un aliment de complément inadéquat. Cependant les bouillies uniquement à base de sorgho sont utilisées par 33,9\% des ménages enquêtés. Bien qu'elles puissent significativement contribuer à l'amélioration de la qualité des aliments infantiles, on se rend compte que les légumineuses participent peu à l'élaboration des bouillies des enfants. Seulement $7 \%$ des ménages enquêtés utilisent les farines composées comme aliment de complément. L'effet significatif du trempage, du tamisage après mouture et de la fermentation sur les composés phénoliques complexes à activité chélateurs comme les tanins témoigne de l'existence de pratiques traditionnelles utiles pour contribuer à l'amélioration de l'état nutritionnel des couches vulnérables. Dans ces conditions de transformation, les facteurs antinutrionnels comme les phytates subissent aussi une dégradation (Kayodé, 2009; Lestiennes, 2004). La valorisation de ces pratiques de transformation au sein des ménages contribuerait à réduire les facteurs antinutritionnels et à améliorer la biodisponibilité des micronutriments pour les 
enfants de 6 à 35 mois. La sensibilisation des mères sur la qualité des aliments qu'elles donnent à leurs enfants favoriserait une utilisation accrue des farines composées et constituerait l'une des solutions locales les plus durables dans la lutte contre la malnutrition infantile. Une fois que les mères seraient convaincues de l'avantage nutritionnel des farines composées on pourrait ensuite les inciter à tremper les farines avant leur cuisson. Ceci améliorerait la biodisponibilité des nutriments et la bioactivité des bouillies consommées.

\section{Conclusion}

Cette étude a permis d'évaluer l'importance du sorgho dans les aliments de complément dans la commune de Natitingou. La composition nutritionnelle de différents types de sorgho a été évaluée. L'effet des opérations unitaires sur la teneur en phénols totaux et en anthocyanes de ces bouillies. De manière générale on note une différence dans la composition nutritionnelle des bouillies étudiées. Seules les bouillies composées présentent un intérêt nutritionnel pour les enfants de 6 à 35 mois. Elles ont cependant une teneur en lipides inférieure aux apports recommandés pour cette tranche d'âge. Le nettoyage des grains de sorgho, le trempage, le tamisage et la fermentation sont des opérations qui réduisent de façon significative la teneur en phénols totaux et en anthocyanes dans la bouillie dérivées de sorgho.

\section{REMERCIEMENTS}

Cette étude a été réalisée dans le cadre du projet INSTAPA, financé par l'Union Européenne [Fp7/2007-2013] sous le numéro d'agrément 211484.

\section{REFERENCES}

AACC. 1984. Approved Methods of the American Association of Cereal Chemistry American Association of Cereal Chemistry. St. Paul MN1.
Abdel-Aal ESM, Hucl P. 1999. A rapid method for quantifying total anthocyanins in blue aleurone and purple pericarp wheats. Cereal Chemistry, 76: 350 - 354.

Black RE, Allen LH, Bhutta ZA, Caulfield LE, de Onis M, Ezzati M, Mathers C, Rivera J. 2008. Maternal and child undernutrition: global and regional exposures and health consequences. Maternal and Child Undernutrition 1, Series. The Lancet, 371: 243-60.

De Benoist B. 1995. Le sevrage un défi pour l'enfant et sa mère. In L'Alimentation de Complément du Jeune Enfant, Trèche $\mathrm{S}$, de Benoist B, Delpeuch F (eds). OSTROM: Paris; 7-13.

Dicko MH, Gruppen H, Traoré AS, Voragen AGJ, Berkel WJH. 2005. Sorghum grain as human food in africa: relevance of content of starch and amylase activities. African Journal of Biotechnology, 5: 384-395.

FAO/OMS. 1994. Codex Alimentarius (Vol. 4, $2^{\text {ème }}$ édn). FAO: Rome, Italie.

FAO/WHO. 2002. Codex Alimentarius. Report of the $24^{\text {th }}$ session of the Codex committee on nutrition and foods for special dietary uses. 4-8 November. Berlin, Germany.

GdB, INSAE, PAM, UNICEF, FAO. 2009. Analyse Globale de la Vulnérabilité, de la Sécurité Alimentaire et de la Nutrition (AGVSAN). République du Bénin (données de novembre et décembre 2008).

Hooper L, Cassidy A. 2006. A review of the health care potential of bioactive compounds. Journal of the Science of Food and Agriculture, 86: 1805-1813.

INSAE, ORC MACRO, USAID, UNICEF. 2006. Troisième Enquête Démographique et de Santé du Bénin (EDSB-III). ORC Macro, Calverton: USA.

Kayodé, APP, Hounhouigan DJ, Nout MJR. 2007. Impact of brewing process operations on phytate, phenolic compounds and in vitro solubility of iron and zinc in opaque sorghum beer. Food Science and Technology/LWT, 40:834-84. 
Kayodé APP. 2006. Diversity, user's perception and food processing of sorghum implications for dietary iron and zinc supply. Ph.D. thesis, Wageningen university, Wageningen, The Nertherlands, p.152.

Kayodé APP. 2009. Biodisponiblilité du fer et du zinc dans les aliments à base de Sorgho. In Recherche et Alimentation en Dialogue, Schärer-Züblin V(ed). Wiley-Blackwell; 274-276.

Latham MC. 2001. La Nutrition dans les Pays en Développement. FAO: Rome, Italie.

Lestienne I. 2004. Contribution à l'étude de la biodisponibilité du fer et du zinc dans le grain de mil et conditions d'amélioration dans les aliments de complément. Thèse de Doctorat, université Montpellier II, Montpellier, France, p. 229.

Mitchikpè EC. 2007. Towards a food-based approach to improve iron and zinc status of rural Beninese children: enhancing mineral bioavailability from sorghum-based food. Ph.D. thesis, Wageningen university, Wageningen, The Nertherlands, p.151.

Nout MJR. 1994. Fermented foods and food safety. Food Research International, 27: 291-298.

Nout MJR, Rombouts FM, Havelaar A. 1989. Effect of accelerated natural lactic fermentation of infant food ingredients on some pathogenic microorganisms. International Journal of Food Microbiology, 8: 351-361.
Nout MJR, Hautvast JGAJ, van der Haar F, Marks WEW, Rombouts FM. 1988. Formulation and microbiological safety of cereal-based weaning foods. In Improving Young Childfeeding in Eastern and Southern Africa, Alnwick D, Moses S, Schmidt OG (éds). Centre de Recherche pour le Développement International: Nairobi, Kenya; 245-260.

Podsędek A, Sosnowska D, Redzynia M, Koziolkiewicz M. 2008. Effect of domestic cooking on the red cabbage hydrophilic antioxidants. International Journal of Food Science and Technology, 43: 1770-1777.

Sandstead HH. 2000. Causes of iron and zinc deficiencies and their effects on brain. Journal of Nutrition, 130: 374-394.

Tulipani S, Mezzetti B, Capocasa F, Bompadre S, Beekwilder J, Rice de Vos $\mathrm{CH}$, Capanoglu E, Bovy A, Battino M. 2008. Antioxidants, phenolic compounds, and nutritional quality of different strawberry genotypes. Journal of Agricultural and Food Chemistry, 56: 696-704.

UNICEF. 2006. Nutrition: le Fer. UnicefFrance: France.

Victora CG, de Onis M, Hallal PC, Blössner M, Shrimpton R. 2010. Worldwide timing of growth faltering: revisiting implications for interventions. Pediatrics, 125(3): e473-e480.

You D, Wardlaw T, Salama P, Jones G. 2010. Levels and trends in under-5 mortality, 1990-2008. The Lancet, 375: 100-103. 